\title{
Applying CLP to Predict Extra-Functional Properties of Component-Based Models
}

\author{
Olivier Defour, Jean-Marc Jézéquel, and Noël Plouzeau \\ INRIA-Rennes, Campus universitaire de Beaulieu, \\ Avenue du général Leclerc 35042 Rennes Cedex, France \\ \{olivier.defour, jean-marc.jezequel, noel.plouzeau\}@irisa.fr
}

\begin{abstract}
A component is the basic re-usable unit of composition to build composite systems by connecting to others through their provided and required ports. Checking the functional compliance between provided and required ports is necessary to build functional systems. At the same time, one of the most important issues today in Component-Based Software Engineering (CBSE) is the prediction of the composite structure Quality of Service (QoS) at design time, using the extrafunctional properties of its components. This paper focuses on this specific CBSE issue, and the use of Constraint Logic Programming (CLP) in this context. For each component providing and requiring services, we propose to specify the QoS properties as required and provided operations, called dimensions, on the component ports. In this model, a QoS property can depend on other QoS attributes, and be constrained by OCL pre- and post-conditions. From this model, the QoS aspect of a component is translated into a QoS system of non-linear constraints over the reals: the dimensions and their pre/post-conditions as variables controlled by nonlinear constraints. These constraints are either inequalities that bound the admissible QoS values, or non-linear functions that bind QoS properties between them. Using the CLP, we are able to determine if a QoS system can be satisfied, and to predict what quality level is required by the assembly from its environment, as a set of admissible intervals. The CLP is a general framework that can be implemented with a realistic effort, to reason about the component-based models QoS properties at design time, that is one of the most important issues in CBSE.
\end{abstract}

\section{Introduction}

The Component-Based Software Engineering (CBSE) community considers any software system as an assembly of components, and promotes methods, technologies and tools in this sense. Software components enable practical reuse: building new solutions by combining bought and home made components improves quality and supports rapid development [4].

Specifications languages for component-based models are yet available, such as UML2.0. However, these models focus only on the functional aspects: the required and provided services, and the connections between components. The 
extra-functional aspect, also called Quality of Service (QoS), is another important part of component-based specifications. This aspect has specific issues, such as:

- monitoring of the QoS properties;

- reasoning about the QoS of an assembly [3].

This paper presents the interest of constraint logic programming over the real $(\mathrm{CLP}(\mathrm{R}))$ applied to this last issue.

\section{Reasoning on the QoS of an Assembly}

A QoS property, also called a dimension [2], is a valuable quantity. Its type is a totally ordered set, such as integer, real, or low; medium; high. Moreover, dependency relationships exist between the dimensions of provided and required service. In order to take into account these properties, we propose to model dimensions as operations, and QoS contracts (set of dimensions) as interfaces [1]. The required or provided levels of quality can be expressed using OCL pre/post conditions.

This metamodel extends usual UML2.0 metaclasses only, and so it is easy to implement into case tool. A model transformation translates the QoS aspect of a QoS-aware component-based model into CLP(R) code where: (1) the components are first order predicates which their arguments are dimensions, constrained in the body by the pre/post conditions, (2) the connections and compositions are conjunctions of component predicates.

\section{Conclusion}

The $\operatorname{CLP}(\mathrm{R})$ is a general framework to handle and reason about the QoS of component-based models. It checks the (QoS) validity of an assembly, and computes an approximated levels of quality. In future works, the model transformation will take into account behavior models, and QoS mutant operators will be developed to (QoS) test a component.

\section{References}

1. Defour O., Jézéquel J.M., Plouzeau N. Extra-functional contracts support in components Component Based Software Engineering (CBSE7), Edinburgh, May 2004.

2. Frolund S. and Koistinen J. QoS specification in distributed object systems Distributed Systems Engineering, vol. 5, July 1998, The British Computer Society

3. Stafford J. and Scott H. The Software Engineering Institute's Second Workshop on Predictable Assembly: Landscape of compositional predictability SEI Report CMU/SEI-2003- TN-029, 2003.

4. Szyperski C. Component software, beyond object oriented programming 2nd ed., Addison-Wesley, 2002 